\title{
DECISION METHODS IN THE THEORY OF ORDINALS ${ }^{1}$
}

\author{
BY J. RICHARD BÜCHI
}

\author{
Communicated by D. Scott, May 21, 1965
}

For an ordinal $\alpha$, let $\operatorname{RS}(\alpha)$, the restricted second order theory of $[\alpha,<]$, be the interpreted formalism containing the first order theory of $[\alpha,<]$ and quantification on monadic predicate variables, ranging over all subsets of $\alpha$. For a cardinal $\gamma, \operatorname{RS}(\alpha, \gamma)$ is like $\operatorname{RS}(\alpha)$, except that the predicate variables are now restricted to range over subsets of $\alpha$ of cardinality less than $\gamma . \omega=\omega_{0}$ and $\omega_{1}$ denote the first two infinite cardinals. In this note I will outline results concerning $\operatorname{RS}\left(\alpha, \omega_{0}\right)$, which were obtained in the Spring of 1964 (detailed proofs will appear in [8]), and the corresponding stronger results about $\operatorname{RS}\left(\alpha, \omega_{1}\right)$, which were obtained in the Fall of 1964.

The binary expansion of natural numbers can be extended to ordinals. If $x<2^{\alpha}$, let $\phi x$ be the finite subset $\left\{u_{1}, \cdots, u_{n}\right\}$ of $\alpha$, given by $x=2^{u_{1}}+\cdots+2^{u_{n}}, u_{n}<\cdots<u_{1} . \phi$ is a one-to-one map of $2 \alpha$ onto all finite subsets of $\alpha$. Let Exy stand for $(\exists u)\left[x=2^{u} \wedge u\right.$ $\in \phi y]$, and note that the algorithm $i+j=s$, for addition in binary notation can be expressed in $\operatorname{RS}\left(\alpha, \omega_{0}\right)$. It now is easy to see that the first order theory FT $\left[2^{\alpha},+, E\right]$ is equivalent to $\operatorname{RS}\left(\alpha, \omega_{0}\right)$, in the strong sense that the two theories merely differ in the choice of primitive notions; the binary expansion $\phi$ yields the translation. Similarly, $\mathrm{RS}(\alpha, \gamma)$ can be reinterpreted as a first order theory. We will state our results in one of the two forms, and leave it to the reader to translate.

TheOREM 1. For any $\alpha$, there is a decision method for truth of sentences in $R S\left(\alpha, \omega_{0}\right)$. The same sentences are true in $R S\left(\alpha, \omega_{0}\right)$ and $R S\left(\beta, \omega_{0}\right)$, if and only if, $\alpha=\beta<\omega^{\omega}$ or else $\alpha, \beta \geqq \omega^{\omega}$ and have the same $\omega$-tail.

If $\alpha=z+\omega^{y}+\omega^{n} c_{n}+\cdots+\omega^{0} c_{0}, y \geqq \omega$, then $z+\omega^{y}$ is called the $\omega$ head of $\alpha$, and $\omega^{n} c_{n}+\cdots+\omega^{0} c_{0}$ is called the $\omega$-tail of $\alpha$.

THeOREM 2. For any ordinals $\beta>\alpha>\omega^{\omega},\left[2^{\beta},+, E\right]$ is an elementary extension of $\left[2^{\alpha},+, E\right]$, if and only if, $\alpha$ and $\beta$ have the same $\omega$-tail. The elementary embedding is then given by $h\left(2^{\alpha 0} x+y\right)=2^{\beta_{0}} x+y$, whereby $x<2^{\tau}, y<2^{\alpha 0}, \tau$ is the common $\omega$-tail of $\alpha$ and $\beta, \alpha_{0}$ and $\beta_{0}$ are respectively the $\omega$-heads of $\alpha$ and $\beta$.

1 This work was supported in part by grant GP-2754 from the National Science Foundation. 
Let $\alpha=\alpha_{0}+\tau \geqq \omega^{\omega}$, where $\alpha_{0}$ is the $\omega$-head and $\tau$ is the $\omega$-tail of $\alpha$. From Theorem 2 one easily shows: the ordinals definable in $\mathrm{FT}\left[2^{\alpha},+, E\right]$ (in FT $\left[2^{\alpha},+\right]$ ) are those of form $2^{\alpha} x+y$, whereby $x<2^{\tau}$ and $y<2^{\left(\omega^{\omega}\right)}$. Actually, Theorems 1 and 2 are but samples of corollaries to Theorem 3 , which completely describes the relations on ordinals definable in FT $\left[2^{\alpha},+, E\right]$.

The results on definability of individuals in $\operatorname{FT}\left[\omega^{\alpha},+\right]$ have been obtained earlier by A. Ehrenfeucht [6]. His methods are quite different; a lucid presentation of this work occurs in [3]. In [3] and [4] it is stated that Ehrenfeucht also knew a decision method for FT $\left[\omega^{\alpha},+\right]$. However, it seems that nobody has checked out these ideas. The first published proof of the decidability of $\mathrm{FT}[\omega,+, E]$, i.e., of $\operatorname{RS}(\omega, \omega)$ occurs in [1], and a similar one in [7]. These are both based on my conjecture that $\operatorname{RS}(\omega, \omega)$ is just strong enough to express the behavior of finite automata.

The key to the understanding of $\operatorname{RS}\left(\alpha, \omega_{0}\right)$ is a natural extension of deterministic finite-state recursions to the transfinite. Let $I$ (input states) and $S$ (internal states) be finite sets. An automaton $\mathfrak{A}$ on $I, S$ consists of an element $A \in S$ (initial state) a map $H: S \times I \rightarrow S$, a map $U: 2^{S} \rightarrow S$, and a subset $0 \subseteq S$ (the output). Let $\sup _{t<x}(r t)$ stand for the set of all values which the function $r$ takes on cofinal to $x$, i.e. $Y \in \sup _{t<x}(r t) \cdot \equiv \cdot(\forall z)_{0}^{x}(\exists t)_{z}^{x}[r t=Y] .[A, H, U]$ determines recursively an operator $s[o, \alpha]=\zeta i[o, \alpha)$ from $I^{\alpha}$ to $S^{\alpha+1}$, namely,

$$
\begin{aligned}
s o & =A, \\
s(x+1) & =B[s x, i x], \\
s x & =U\left[\sup _{i<x}(s t)\right], \quad x \text { a limit. }
\end{aligned}
$$

An input sequence $i[o, \alpha)$ is said to be accepted by $\mathfrak{A}$, in case $s \alpha \in 0$. Extending the proofs given in [1], one now shows,

TheOREM 3. Let $R\left(i_{1}, \cdots, i_{n}\right)$ be a relation on finite predicates on $\alpha$. $R$ is definable in $R S\left(\alpha, \omega_{0}\right)$ if and only if there is an automaton $\mathfrak{A}$ such that $R$ consists of those finite $\left(i_{1}, \cdots, i_{n}\right)$ on $\alpha$, for which the input signal $i[0, \alpha)$ is accepted by $\mathfrak{A}$.

In fact there are effective methods, (1) for the construction of $\mathfrak{A}$ from a defining formula $\Sigma$ of $R$ (synthesis), and (2) for the construction of $\Sigma$ from $\mathfrak{A}$ (analysis). Theorems 1 and 2 now follow by investigating the behavior of input-free automata.

Let us now consider $\operatorname{RS}\left(\alpha, \omega_{1}\right)$. The decidability of $\operatorname{RS}\left(\omega_{0}, \omega_{1}\right)$, i.e., $\mathrm{RS}\left(\omega_{0}\right)$ was proved in [2]. It is not difficult to extend the method used 
in [2], replacing ordinary automata recursions by transfinite automata. The result is,

Theorem 1'. For any countable ordinal $\alpha, R S(\alpha)$ is decidable. For $\alpha<\beta<\omega_{1}, R S(\alpha)$ and $R S(\beta)$ are equivalent if and only if either $\alpha=\beta$ $<\omega^{\omega}$ or $\alpha, \beta \geqq \omega^{\omega}$ and have the same $\omega$-tail. Furthermore, $R S\left(\alpha, \omega_{1}\right)$ is decidable for any $\alpha$.

As in [2] we actually obtain a complete survey over definability in $\operatorname{RS}\left(\alpha, \omega_{1}\right)$. In particular, the analog to Theorem 2 holds.

Define the $\alpha$-behavior of an automaton $\mathfrak{A}$ to be the set $\operatorname{Bh}(\mathfrak{A}, \alpha)$ consisting of all input-signals $i[, o \alpha)$ which are accepted by $\mathfrak{A}$. Thus, the $\omega$-behaviors are the ordinary regular sets of finite automata theory.

THEOREM 4. To any automaton $\mathfrak{A}$ with input $(i, j)$ one can construct an automaton $\left(\mathbb{S}\right.$ with input $i$, such that for any $\alpha \leqq \omega_{1}$ and any inputsignal $i$ of length $\langle\alpha, i \in B h(\mathfrak{E}, \alpha) \cdot \equiv \cdot(\exists j)(i, j) \in B e h(\mathfrak{A}, \alpha)$.

For $\alpha=\omega$ this is the well-known projection-lemma for behaviors of finite automata. The case $\alpha=\omega+1$ constitutes a significant improvement of the crucial Lemma 9 of [2], and has recently been obtained by $\mathrm{R}$. McNaughton. His construction is very ingenious, and his ['s are by far the most intricate finite automata this writer has seen in action. The extension to $\alpha \leqq \omega_{1}$ is an exercise in handling ordinals. Using this improved form of Lemma 9, the definability result of [2] extends as follows,

ThEOREM $3^{\prime}$. To every RS-formula $\Sigma\left(i_{1}, \cdots, i_{n}\right)$ one can construct an automaton $\mathfrak{A}$, and to every automaton $\mathfrak{N}$ with $2^{n}$-ary input $\left(i_{1}, \cdots, i_{n}\right)$ one can construct an $R S$-formula $\Sigma\left(i_{1}, \cdots, i_{n}\right)$, such that for any $\alpha<\omega_{1}$ the behavior $B h(\mathfrak{A}, \alpha)$ is the relation defined by $\Sigma$ in $R S\left(\alpha, \omega_{1}\right)$.

The following problem remains unsolved: Is $\mathrm{RS}\left(\omega_{1}\right)$ decidable?

\section{BIBLIOGRAPHY}

1. J. R. Büchi, Weak second order arithmetic and finite automata, Z. Math. Logik Grundlagen Math. 6 (1960), 66-92.

2. - On a decision method in restricted second order arithmetic, Proc. Int. Cong. Logic, Method. and Philos. Sci., 1960, Stanford Univ. Press, Stanford, Calif., 1962.

3. S. Feferman, Some recent work of Ehrenfeucht and Fraïsse, Summer Institute for Symbolic Logic, Cornell Univ., 1957, Commun. Research Div., Institute for Defense Analyses, 1960, pp. 201-209.

4. S. Feferman and R. L. Vaught, The first order properties of products of algebraic systems, Fund. Math. 47 (1959), 57-103.

5. R. McNaughton, Reviews of Weak second order arithmetic and finite automata 
and $O n$ a decision method in restricted second order arithmetic by J. R. Büchi, J. Symb. Logic 28 (1963), 100-102.

6. A. Ehrenfeucht, Application of games to some problems of mathematical logic, Bull. Acad. Polon. Sci. 5 (1957), 35-37.

7. C. C. Elgot, Decision problems of finite automata design and related arithmetics, Trans. Amer. Math. Soc. 98 (1961), 21-51.

8. J. R. Büchi, Transfinite automata recursions and weak second order theory of ordinals, Proc. Int. Cong. Logic, Method. and Philos. Sci., Jerusalem, 1964 (to appear).

Ohio State University 